\title{
Kinematics of collagen fibers in carotid arteries under tension-inflation loading
}

\author{
Witold Krasny ${ }^{1,2,3,4}$. Hélène Magoariec ${ }^{4}$. \\ Claire Morin $^{1,2,3}$. Stéphane Avril ${ }^{1,2,3 *}$
}

Received: date / Accepted: date

\begin{abstract}
Biomechanics of the extracellular matrix in arteries determines their macroscopic mechanical behavior. In particular, the distribution of collagen fibers and bundles plays a significant role. Experimental data showed that in most arterial walls there are preferred fiber directions. However, the realignment of collagen fibers during tissue deformation is still controversial: whilst authors claim that fibers should undergo affine deformations, others showed the contrary. In order to have an insight about this important question of affine deformations at the microscopic scale, we measured the realignment of collagen fibers in the adventitia layer of carotid arteries using multiphoton microscopy combined with an unprecedented Fourier based method. We compared the realignment for two types of macroscopic loading applied on arterial segments: axial tension under constant pressure (scenario 1) and inflation under constant axial length (scenario 2). Results showed that, although the tissue underwent macroscopic stretches beyond 1.5 in the circumferential direction, fiber directions remained unchanged during scenario 2 loading. Conversely, fibers strongly realigned along the axis direction for scenario

1. Ecole Nationale Supérieure des Mines de Saint-Etienne, CIS-EMSE, SAINBIOSE, F-42023 Saint Etienne, France

2. INSERM, U1059, F-42000 Saint Etienne, France

3. Université de Lyon, SAINBIOSE, F-42000 Saint Etienne, France

4. Laboratoire de Tribologie et Dynamique des Systèmes, CNRS UMR 5513, Université de Lyon, Ecole Centrale Lyon, France

*correspondance to: avril@emse.fr
\end{abstract}


1 loading. In both cases, the motion of collagen fibers did not satisfy affine deformations, with a significant difference between both cases: affine predictions strongly under-estimated fiber reorientations in uniaxial tension and over-estimated fiber reorientations during inflation at constant length. Finally, we explained this specific kinematics of collagen fibers by the complex tension-compression interactions between very stiff collagen fibers and compliant surrounding proteins. A tensegrity representation of the extracellular matrix in the adventitia taking into account these interactions was proposed to model the motion of collagen fibers during tissue deformation.

Keywords Carotid artery · tension-inflation · collagen · adventitia · fiber bundles · reorientation $\cdot$ kinematics

\section{Introduction}

The arterial tissue exhibits a hierarchical microstructure, made of three concentric layers, namely, starting from the arterial lumen towards the external surface of the vessel: the intima, the media, and the adventitia. The extracellular matrix of these three layers is made of arrangements of collagen and elastin fiber networks, with a specific organization depending on the layer. In particular, the non-linear mechanical behavior of the arterial tissue is often associated with the progressive rearrangements of these different fiber networks, at the 10 to $100 \mu \mathrm{m}$ scale. In the present contribution, we chose to focus on the adventitial collagen, since it was recently shown to be the fiber network the most prone to mechanically-induced rearrangements $[44,12,49,31]$. In particular, this network, composed of thick bundles of type I collagen fibers [15], is the major structural component of the adventitia, due to its important load-bearing function preventing over-distensions [26]. Eventually, its remodeling under pathological situations such as aneurysms may degrade this load bearing function [27].

Various experimental loading setups coupled to live microscopy provided a better understanding of the fiber kinematics, allowing progressive improvements of 
structural constitutive models of the arterial wall. Namely, in the unloaded configuration, the adventitial collagen network shows randomly distributed orientations and a high degree of crimping $[15,39,41]$. Upon mechanical loading, the collagen bundles progressively unfold $[8,21]$. The measurement of fiber waviness $[11,44,53$, 49] revealed in particular that recruitment, or engagement of collagen fibers, is gradual (unsynchronized among fibers) and starts at a finite strain $[23,13]$. The process can differ whether the observed vascular region is close or remote from the heart (distal regions vs. proximal regions) [55]. Collagen fiber bundles may also undergo a progressive realignment in response to the application of a macroscopic deformation $[1,30,11,13,31]$. This realignment has been observed to take place after recruitment, in order for the artery to continue deforming without damaging the engaged fibers [53], and showed discrepancies in fiber realignment amplitudes, depending on the initial fiber angle and on the type of deformation [52,12]. The fiber bundles are finally stretched upon further mechanical loading. Eventually, rupture of the collagen fibers may lead to the rupture of the adventitial layer. Consequently, a correct assessment of the mechanical fields within the adventitia relies on a correct description of the collagen network and of its evolution during loading.

The aforementioned experimental findings enhanced the first generation of structural models based on the definition of a strain energy function, which considered the arterial wall as a soft matrix material reinforced by variously distributed fibers families $[24,58,25,19]$, with the addition of a fiber activation stretch or of a probability distribution function, accounting for gradual recruitment at finite strain $[42,23]$. All these models assume an affine deformation of the collagen network, as experimentally evidenced by several contributions [43,52], i.e. the fibers are assigned to follow the overall material deformation: they are firmly embedded in the surrounding matrix. The resulting understanding of collagen kinematics and its integration in structural models provides today satisfactory predictions of the artery's mechanical response $[58,25]$. However, questions remain about the 
microscopic mechanisms governing the observed rearrangements. The latter question is especially raised with regards to past studies, revealing non-affine collagen kinematics $[5,10,29,31]$, illustrating the complexity of arterial microstructure properties. Whether they are attributed to the decrimping process [34], to fiberfiber interactions [13] or to fiber-matrix interactions [5,50], non-affine collagen kinematics are currently taken into account in a novel generation of multiscale and micromechanical modelling approaches $[48,16,56,37]$, but their microstructural and micromechanical origin has not yet been experimentally documented nor understood, which constitutes a limit for further refined multi-scale formulations. Specifically, few studies investigate the dependence of fiber kinematics on the loading type $[52,12]$, and to the authors' knowledge, none have quantitatively compared fiber rotations for the same tracked fibers undergoing different loading scenarii, while comparing them to affine predicted reorientations. In the present study, we bridge this gap by answering the following questions: do particular deformation scenarii or conditions challenge affinity of fiber motion? Is the fibers behavior different under the different loading scenarii? To this aim, we submitted carotid arteries from New Zealand White rabbits to tension-inflation tests, while quantitatively characterizing the reorientations of its adventitial collagenous microstructure with multi-photon microscopy.

\section{Materials and methods}

2.1 Sample preparation

Carotid arteries $(\mathrm{n}=4)$ were harvested at the Veterinary Campus of the Université de Lyon (VetAgro Sup, Marcy l'Étoile, FR) from healthy male New Zealand White rabbits, weighing $3 \mathrm{~kg}$ approximately. The cadavers, kindly provided by Centre Lago (Vonnas, FR), were previously sacrificed under compliance with the NIH Guide for Care and Use of Laboratory Animals. Immediately after excision, the samples were stored in a bath of phosphate-buffered saline (10x PBS, pH 7.1) 
at $5^{\circ} \mathrm{C}$ and tested within 36 hours after harvesting. For each sample, a $15 \pm 1 \mathrm{~mm}$ long cylindrical segment was extracted for mechanical testing (Fig. 1(a), left image) as well as three $0.5 \mathrm{~mm}$ long rings for optical measurement of the sample thickness (Fig. 1(a), right image). The sample tips were cannulated onto the inlet and outlet needles using suture wire (Fig. 1(a)). Two plastic rings previously fixed on the needles at the suture loci (Fig. 1(f)) prevented slipping during axial tension and provided a waterproof sealing during inflation. The sealing rings provided structural support to sustain axial tension and no damage was observed on the samples at the locus of the ring after the mechanical tests.

\subsection{Mechanical setup}

We hereafter describe the mechanical setup that enabled tension-inflation of the carotid arteries. Applying an axial tension was made possible by a screw-driven high precision tensile machine (Deben ${ }^{\circledR}$ Microtest tensile/compression stage) equipped with a $150 \mathrm{~N}$ load cell (Fig. 1(b) and 1(e)) which provided a $0.01 \mathrm{~N}$ precision with satisfactory stability during the tests. The two heads of the machine moved in opposite directions, allowing the operator to identify and keep track of a region of interest, while the microscope was positioned at the center of the sample. The force offset was zeroed prior to the mounting of the sample. Verifying that the axial force and sample length were unchanged after mounting allowed limiting the risk of potential pre-strain and pre-stress in the sample. A pressure loading was applied by a syringe pump (Fig. 1(c)) (Harvard Apparatus ${ }^{\circledR}$ ) equipped with a $\pm 300 \mathrm{mmHg}$ pressure transducer $\left(\right.$ FISO $^{\circledR}$ optical fiber connected to the fluid network at the sample inlet) which infused phosphate-buffered saline (10x PBS, $\mathrm{pH}$ 7.1) at a constant ambient temperature of $20^{\circ} \mathrm{C}$. Applying a target pressure was made possible by the pump and pressure transducer communicating under closed loop control (PID mode). The settings of the proportional gain and integrator constant were iteratively adjusted in order to obtain a stable pressure step, characterized by a reasonable pressurizing time and limited overshooting. During 
the mechanical loading, the sample was continuously immerged in PBS at a constant ambient temperature of $20^{\circ} \mathrm{C}$ (Fig. 1(b)). Also, the pressure readouts at inlet and outlet were compared to ensure that the pressure was uniformly distributed and did not vary due to friction forces in the needles during infusion. An optical camera (Nikon ${ }^{\circledR}$ D7200 equipped with Nikon ${ }^{\circledR}$ AF-S VR Micro-Nikkor optical $105 \mathrm{~mm} \mathrm{f} / 2.8 \mathrm{G}$ IF-ED lens) was used to acquire images of the deforming arterial sample and of the three sample rings, for subsequent analysis of sample diameter and sample thickness (Fig. 1(a)). Noticeably, the measurement of the arteries' diameter showed a limited variability along the sample length (standard deviations being as low as $2 \%$ of the measured mean diameters).

\subsection{Multiphoton Microscopy}

The multiphoton microscope (NIKON, A1R MP PLUS ${ }^{\circledR}$ ) of the IVTV platform (Engineering and Ageing of Living Tissues Platform, ANR-10-EQPX-06-01, Lyon, FR) was used to image the fibrous microstructure of the tested arterial segments (Fig. 1(d)), without prior staining or fixation. Using a $870 \mathrm{~nm}$ excitation wavelength [23], the collagen second harmonic generation ( $\mathrm{SHG}$ ) signal was acquired through 400-492 $\mathrm{nm}$ band-pass filters. The multiphoton imaging modality was well documented in previous studies [51,39,49], should additional information be needed. The imaging resolution of the microscope was set to $0.5 \mu \mathrm{m}$ in all directions $[23,44]$ with a $512 \mu^{2}$ imaging window. An optimal compromise was achieved between image quality and acquisition time by setting the scan speed to 0.5 frames per second, with two-frame averaging. These setting resulted in 60 to 90 mm thick stacks of images with acquisition times of approximately 15 minutes. A period of 5 minutes was always observed after applying an incremental load step to the artery in order to stabilize the tissue before imaging it. However, this apparent creeping occurring after each load step applied on the arterial tissue did not change the microstructural morphology of the tissue, as observed by live imaging. 


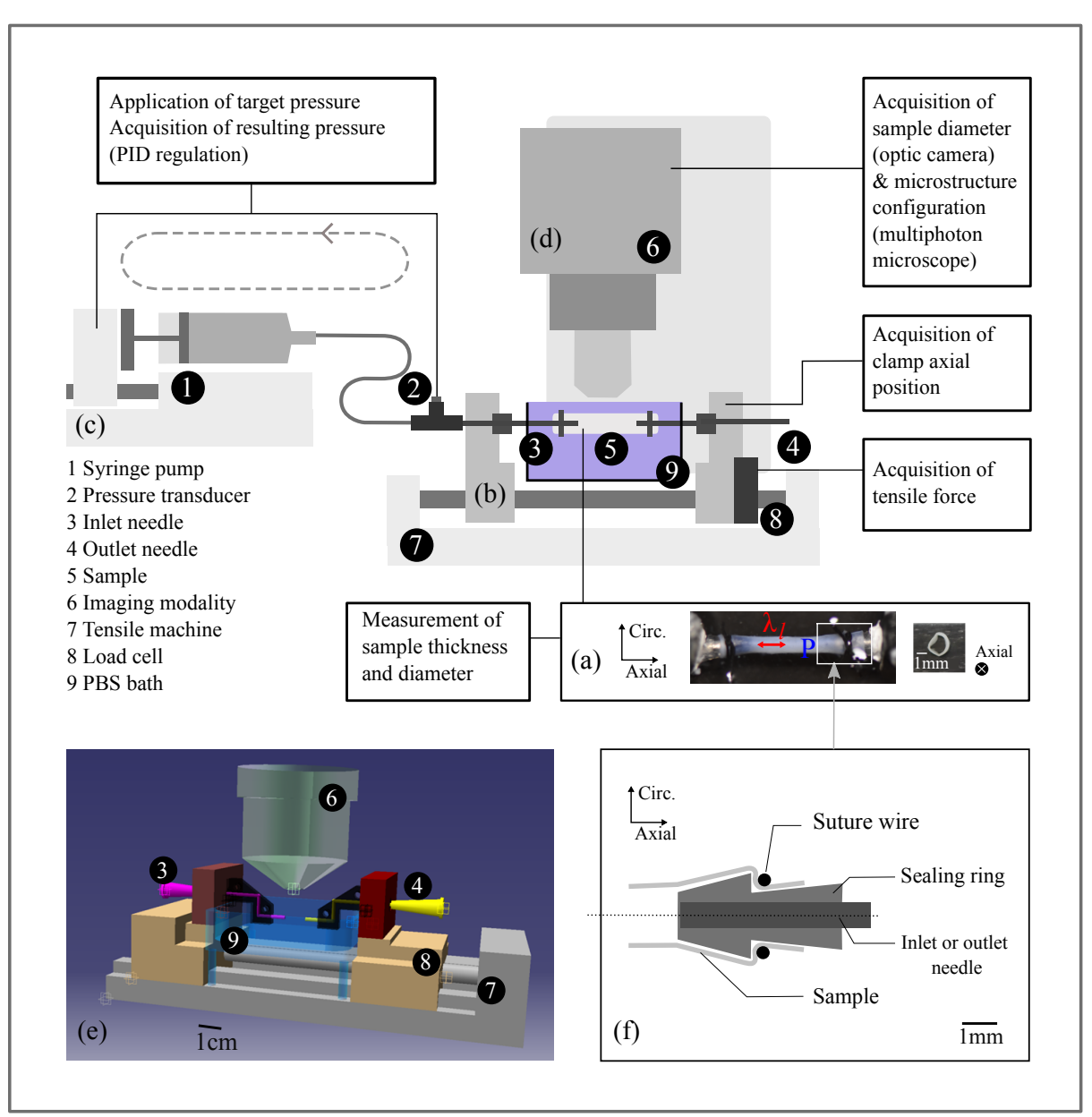

Fig. 1 Experimental tension-inflation setup showing (a) a sample cannulated on the needles and loaded, comprising of (b) the tensile machine, (c) the syringe pump, (d) imaging modalities (alternatively optical camera and multiphoton microscope); (e) a schematic representation of the tension-inflation setup, showing the tensile machine, the PBS bath in blue transparency, needle stiffening casings in black transparency, inlet (pink) and outlet (green) needles, the multiphoton microscopes objective in grey (center); and (f) a section sketch of the cannulated arterial sample for tension-inflation testing, presenting the sealing method.

\subsection{Loading scenario and data acquisition}

In agreement with well-established tension-inflation protocols for arteries [26,30], two loading scenarii were considered, namely axial tension under constant imposed pressure and inflation under constant axial stretch. Four carotids were used in the present study, carotid samples S1 and S2 were tested under both loading scenarii, while carotid samples S3 and S4 were tested under only one loading scenario, i.e. 

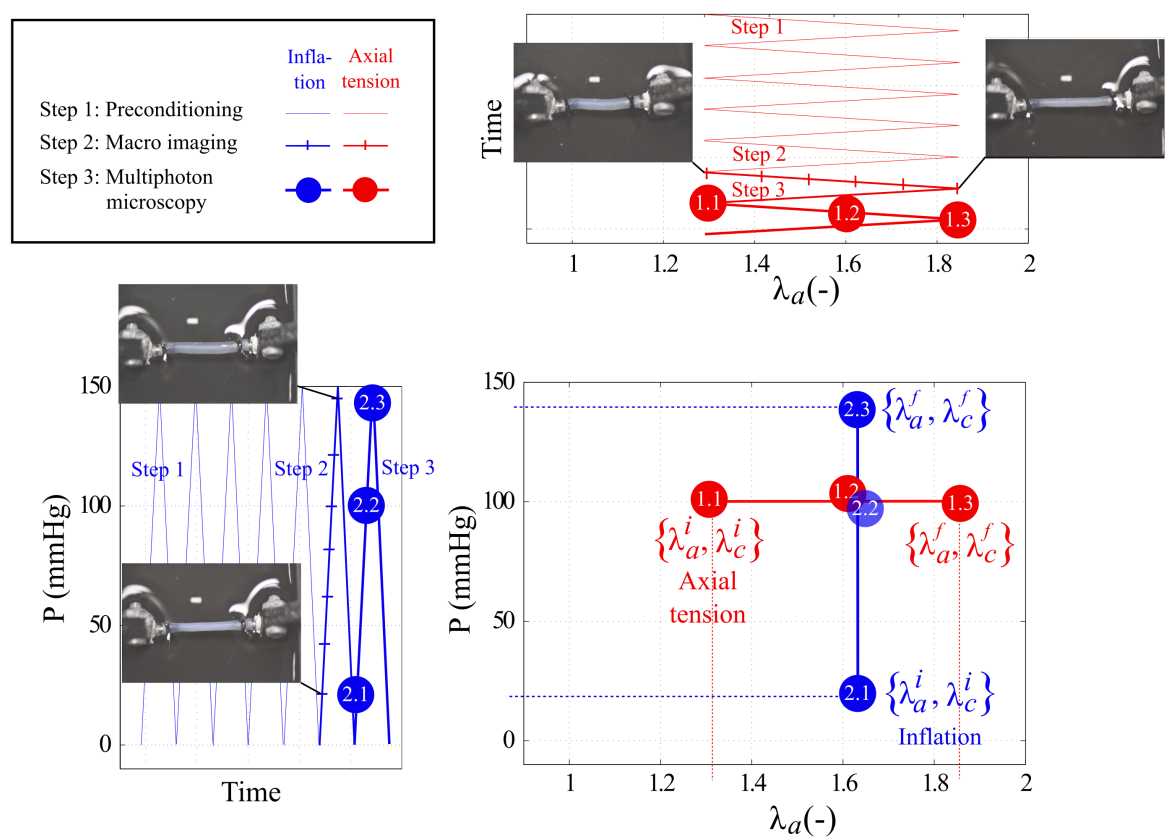

Fig. 2 Tension-inflation loading protocol, comprised of three loading steps for both axial tension and inflation scenario, namely (Step 1) preconditioning (thin line), (Step 2) macroscopic imaging sequence (thick line with ticks denoting macro images), and (Step 3) multiphoton microscopy sequence (thick line with circles denoting loading configurations in which the collagen morphology was acquired). The morphologies referring to load states 1.1, 1.3, 2.1 and 2.3 are used as inputs for subsequent image processing and fiber kinematics characterization (see Section 2.5 and Figure 3). The tensile loading scenario is represented in red, whereas the inflation loading scenario is represented in blue.

the axial tension and the inflation scenario, respectively. For each sample and each loading scenario, the loading protocol was composed of three loading steps (Fig. 2 ). Step 1 consisted in five preconditioning cycles, carried out in order to cancel the tissue's deformation history, step 2 consisted in one loading cycle dedicated to the acquisition of macroscopic sample dimensions, and step 3 consisted in one final loading cycle dedicated to the acquisition of the collagen load-dependent morphologies, by means of multiphoton microscopy. As a result, on the one hand, three samples (S1, S2, and S3) underwent a total of seven axial tension cycles (with the two last cycles dedicated to data acquisition) between $0.1 \pm 0.02$ and $0.8 \pm 0.02 \mathrm{~N}$ axial force, at an imposed velocity of $2 \mathrm{~mm} \cdot \mathrm{min}^{-1}$, corresponding to an axial strain rate of $0.2 \mathrm{~min}^{-1}$, with maintained pressure $(\mathrm{P}=100 \mathrm{mmHg})$. 
On the other hand, three samples (S1, S2, and S4) underwent a total of seven inflation cycles (with the two last cycles dedicated to data acquisition) between 20 and $140 \mathrm{mmHg}$, by steps of $20 \mathrm{mmHg}$ imposed within 30 seconds, under a maintained in vivo axial stretch $\left(\lambda_{a}=1.6 \pm 0.05\right)$. The in vivo stretch, defined as the stretch resulting in unchanged axial reaction force during inflation [26], was determined by adjusting the clamp position during the preconditioning cycles. The samples reference cross-sectional areas $A_{0}$ were computed from the measures of the unloaded sample diameters after preconditioning (Step 1 of the protocol) and from the initial thicknesses. In the case of the axial tension load scenario, the zero axial strain position was obtained from the axial position at which the axial force returned to zero after preconditioning. The measurement of the sample length after preconditioning provided the reference length $l_{0}$ on the basis of which the macroscopic axial stretch was computed as $\lambda_{a}=l / l_{0} . l$ and $l_{0}$ refer to the current and the reference inter-clamp lengths of the sample, respectively. Similarly, in the case of the inflation loading scenario, the zero circumferential strain position was obtained from the measurement of the sample diameter after preconditioning, when the inflation system was opened, forcing the pressure to return to $0 \mathrm{mmHg}$ in the sample. The measurement provided the reference diameter $D_{0}$ on the basis of which the macroscopic circumferential stretch was computed as $\lambda_{c}=D / D_{0} . D$ and $D_{0}$ refer to the current and the reference diameters of the sample, respectively. After preconditioning, the gauge length of the samples (between suture) measured $l_{0}=12 \pm 2 \mathrm{~mm}$, while the unloaded sample diameter measured $D_{0}=2.0 \pm 0.3 \mathrm{~mm}$. Macroscopic images of the samples (Step 2 of the loading protocol) were obtained every $20 \mathrm{mmHg}$ during inflation and every $0.5 \mathrm{~mm}$ during axial tension, corresponding to $0.2 \pm 0.05$ stretching steps approximately (see thick lines with ticks on Fig. 2, showing macroscopic imaging). Microscopy images (Step 3 of the protocol) were obtained at at $1.3 \pm 0.05,1.6 \pm 0.05$, and $1.8 \pm 0.05$ stretches during axial tension (referenced as loading configurations 1.1, 1.2, and 1.3, respectively (Fig. 2), and at $20 \mathrm{mmHg}, 100 \mathrm{mmHg}$, and $140 \mathrm{mmHg}$ pressures during inflation (referenced 
as loading configurations 2.1, 2.2, and 2.3, respectively (Fig. 2)). The mechanical configurations 1.1 and 2.1 will be referred to in the following as "reference configurations", while the mechanical configurations 1.3 and 2.3 will be referred to as "loaded configurations". As concerns the intermediate loading configurations 1.2 and 2.2, they were not used in the Fourier methodology enabling the characterization of fiber kinematics affinity but provided a refined insight at the evolution of the microstructure during the loading (see Fig. 4 in Section 3), and a verification of the right tracking of the same imaging windows fields of view. The reference configurations included partial loading $\left(\lambda_{a}=1.3, \mathrm{P}=100 \mathrm{mmHg}\right.$ for scenario 1, $\lambda_{a}=1.6, \mathrm{P}=20 \mathrm{mmHg}$ for scenario 2) for several reasons. First, we chose to post-process adventitial collagen images characterized by a sufficient degree of decrimping, hence avoiding imprecisions in the Fourier analysis of the global orientations of the fibers (at the bundle scale); second this choice prevented the potential buckling of the samples at high pressure and low axial stretch [22], which could have impaired the analysis of macroscopic and microscopic kinematics.

\subsection{Image analysis and characterization of fiber kinematics}

The previously collected mechanical and microstructural information is now used to analyze the fiber rearrangements and the corresponding fiber kinematics for the different loading scenarii.

Firstly, image stacks corresponding to the reference configurations 1.1 and 2.1 (Fig. 2), as well as image stacks corresponding to the loaded configurations 1.3 and 2.3 (Fig. 2), were processed using a Maximum Intensity Projection (MIP) algorithm (Fig. 3 (a1) and (a2)), revealing optimal in-plan morphology. In fact, previous studies have investigated the transmural angle (radial direction) of the fibers and showed that it is negligible in comparison to the in-plane angle [42, 41,44]. As a result, although the negligible transmurality information is lost, the fibers are represented with maximal effective length, strongly improving the interpretation of their in-plane orientation. A preliminary geometrical analysis revealed 
that the potential evaluation error of in-plane fiber orientation caused by projecting slices of a cylinder into a 2D image, was always under $5 \%$ (maximal at the image periphery, where the tangential plane shows the highest inclination to the projection plane), and hence was considered negligible.

Secondly, the affine transformation was simulated, by numerically applying axial and circumferential deformations to the reference configurations. In more details, for the loading scenario 1 (axial tension under constant pressure), a numerical axial stretch $\lambda_{a}^{*}$ together with the corresponding measured transverse contraction (resulting from diameter change) $\lambda_{c}^{*}$ were gradually applied to the image of the unloaded configuration 1.1 (Fig. 2). Similarly for loading scenario 2 (inflation under constant axial stretch), a numerical circumferential stretch $\lambda_{c}^{*}$ (corresponding to outer diameter change) was gradually applied to the image of the reference configuration 2.1 (Fig. 2). Image resolution remained unchanged by the use of subpixel interpolation.

Afterwards, the 2D Fourier spectrum moduli were extracted from the collagen morphologies for both the numerically deformed (Fig. 3 (c1)) and the experimentally loaded configurations (Fig. $3(\mathrm{c} 2)$ ). Use of the 2D Fourier transforms allowed removing the non-mechanical optical effects (such as contrast or brightness effects) as well as the artifacts (features occurring at short lengthscale, such as the decrimpring process), and the inequality of imaged windows. Previous studies have proven the potential of the Fast Fourier transform (FFT 2D) for the analysis of biomedical images, and in particular the ability of the method to extract fiber angle densities or isotropy parameters $[2,14,45,40,13,38]$. Here a special attention was paid to work with spectra being matrices of identical dimensions (Fig. 3, red cropping square). A preliminary analysis allowed verifying that the locus of the cropping center in the image did not affect the results. Each spectrum was then multiplied by the Fourier transform of a Gaussian window whose size corresponded to the characteristic width of a collagen bundle (1/25 of the imaged window, equivalent to $20 \mu \mathrm{m}$ ), allowing the filtering of undesirable small-length features in the 
images and the highlighting of meaningful morphological features (namely fiber orientations at the scale of the bundles) (Fig. 3 (d)) [57]. The obtained 2D Fourier spectrum moduli $\mathrm{S}^{*}$ and $\mathrm{S}$ (numerically and experimentally deformed microstructures, respectively) were finally compared using a relative norm-2 (Fig. 3 (d)).

We gradually increased the numerically applied affine deformation, eventually reaching a minimum in the relative difference between the two collagen morphologies (i.e. configurations 1.3 or 2.3 and the corresponding affine deformations of configurations 1.1 and 2.1, Fig. 2). As a result, this minimum corresponded to the application of a fictitious stretch that should be applied to the reference microstructure in order to reach by affine deformation the most similar microstructure to the loaded experimental configuration. Eventually, this fictitious stretch was compared to the actual experimentally measured stretch. If the fictitious stretch and the actual stretch are equal, the kinematics at the scale of collagen fibers actually remained affine. 

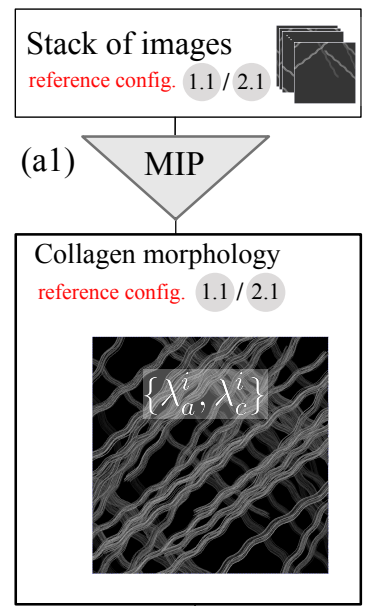

(b) Affine deformation

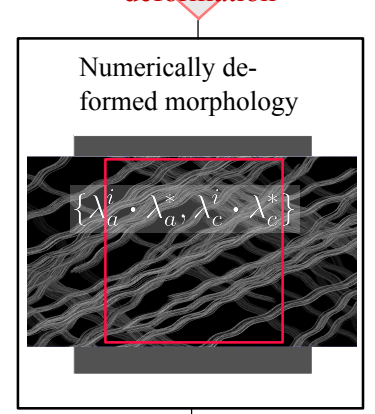

(c1) FFT 2D

2D spectrum modulus
Stack of images

loaded config. $1.3 / 2.3$

(a2)

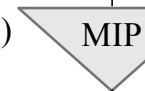

Collagen morphology

loaded config. $1.3 / 2.3$

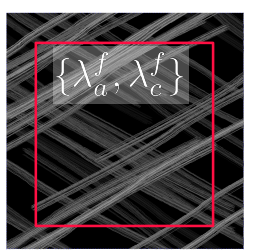

(c2) FFT 2D

2D spectrum modulus

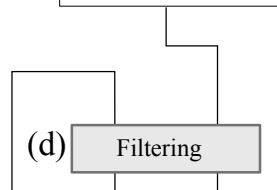

(e) Spectra comparison

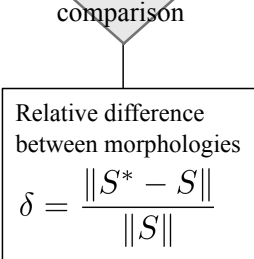

Fig. 3 Series of operations summarizing the image analysis and characterization methodology of fiber kinematics. The methodology consists in (i) analyzing the stacks of images in the reference (a1) and loaded (a2) configurations by means of a Maximum Intensity Projection (MIP); (ii) numerically applying a gradual affine deformation to the reference configurations (b); and (iii) comparing the microstructure configurations through their FFT spectra (c), so as to extract the affine deformation (applied numerically), which produces a microstructure with minimum relative difference between the experimental morphology and the numerically deformed one (d). For each loading scenario, the axial stretch ranges between $\lambda_{a}^{i}$ (reference configuration) and $\lambda_{a}^{f}$ (loaded configuration) $\left(\lambda_{a}^{i}=\lambda_{a}^{f}\right.$ in the case of the inflation loading), whereas the circumferential stretch ranges between $\lambda_{c}^{i}$ (reference configuration) and $\lambda_{c}^{f}$ (loaded configuration). In this figure, the used images are custom-drawn visual representations of collagen morphology, chosen for clarity and representativeness. 


\section{Results}

(a)
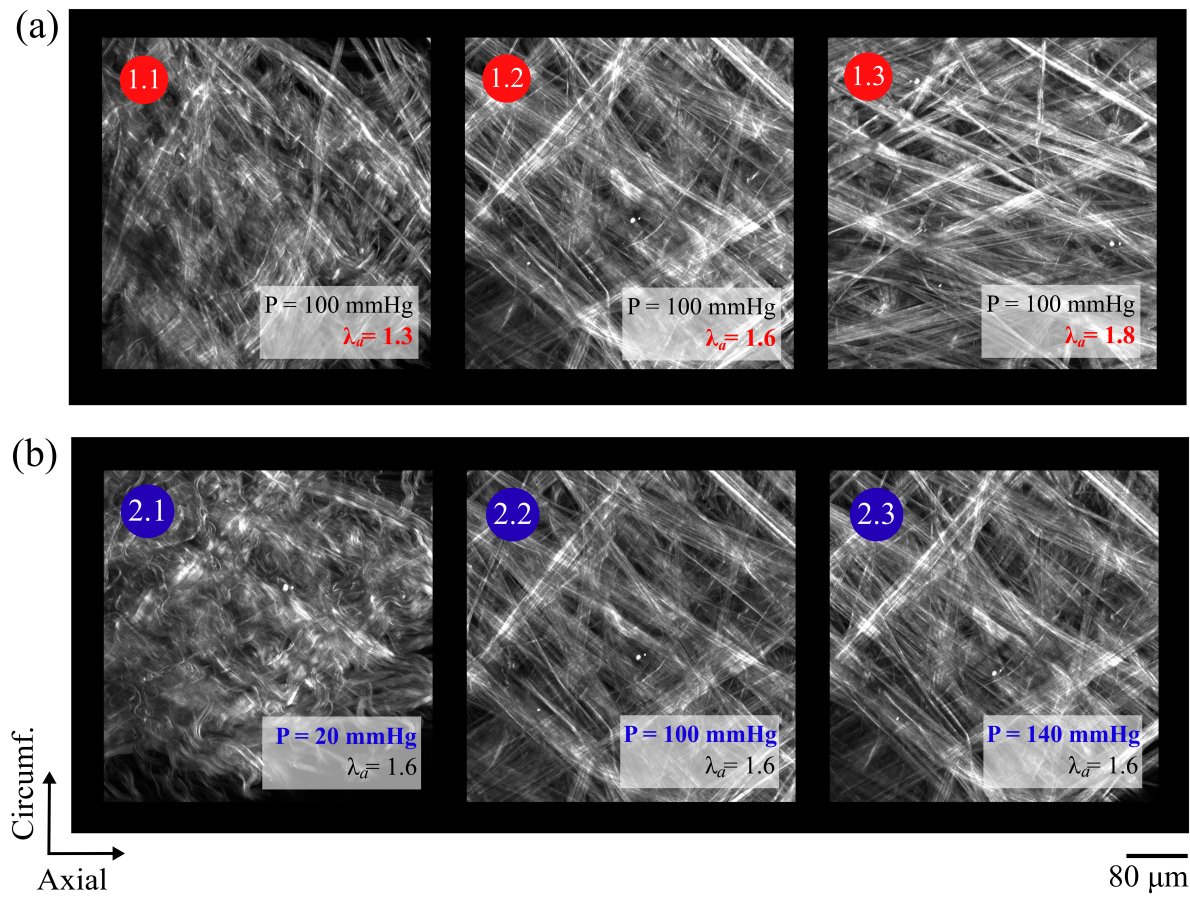

Fig. 4 Adventitial collagen morphology under both loading scenarii, revealed through multiphoton microscopy for sample S1: (a) tension under imposed constant pressure and (b) inflation under imposed constant axial stretch.

First, we qualitatively compared the microstructure evolution between the two loading scenarii (Fig. 4). Fig. 4(a) shows that, under axial tension at a constant intermediate pressure, adventitial collagen fibers achieved full straightening and reoriented towards the macroscopic strain direction. On the contrary, Fig. 4(b) shows that, under inflation at the in vivo stretch, collagen fibers of the adventitia achieved full straightening but the orientation of the majority of the fibers remained unchanged. Similar images, and hence similar analyses were obtained for the 2 other samples in each loading direction. Imaging always the same re- 
gion of interest in the tissue at the different stages of the deformation made the characterization of fiber reorientations reliable.

As a second step, we quantitatively analyzed the kinematics of the fiber rotation for each loading scenario (Fig. 5). For loading scenario 1 (corresponding to axial tension under constant imposed pressure), the difference $\delta$ between the numerical and the experimental morphologies started, for the three samples, at high values: this denotes different microstructure morphologies between the reference arterial configuration and axially loaded arterial configuration, particularly in terms of fiber orientations (Fig. 5(a1)). As the numerically applied affine deformation increased, the fibers were gradually reoriented towards the direction of axial tension, hence coming closer to the experimental fiber orientations of the loaded configuration: the relative difference $\delta$ between the spectra decreased. When the applied affine deformation became too high, the relative difference increased again: affine deformation induced fiber rotations being larger than the ones experimentally observed (Fig. 5(a3)). In between, the relative difference $\delta$ reached a minimum value, when the fibers of the experimentally and the numerically deformed configurations showed the closest fiber orientations (Fig. 5(a2)). For the three tested samples $\mathrm{S} 1, \mathrm{~S} 2$, and $\mathrm{S} 3, \delta$ reached its minimum at axial stretch values comprised between 1.85 and 2.1, i.e. to obtain similar morphologies, the numerically applied stretch needs to be $20 \%$ higher than the experimentally applied stretch (Fig. 5, black dots). For loading scenario $2, \delta$ starts, for the three samples, at very low values: this denotes similar microstructure morphologies between the reference and the loaded arterial configurations, particularly in terms of fiber orientations (Fig. $5(\mathrm{~b} 1))$. As the numerically applied circumferential stretch increased, the fibers reoriented towards the circumferential load direction, hence driving away from the fiber orientations of the experimentally loaded configuration: this led to a continuously increasing relative difference $\delta$ between the spectra ((Fig. 5(b2) and (b3)). For the three tested samples $\mathrm{S} 1, \mathrm{~S} 2$, and $\mathrm{S} 4$, the minimum of $\delta$ was reached at circumferential stretch values comprised between 1.1 and 1.13, i.e. to have com- 
parable morphologies, the numerically applied stretch needs to be $30 \%$ lower than the experimentally applied stretch. 

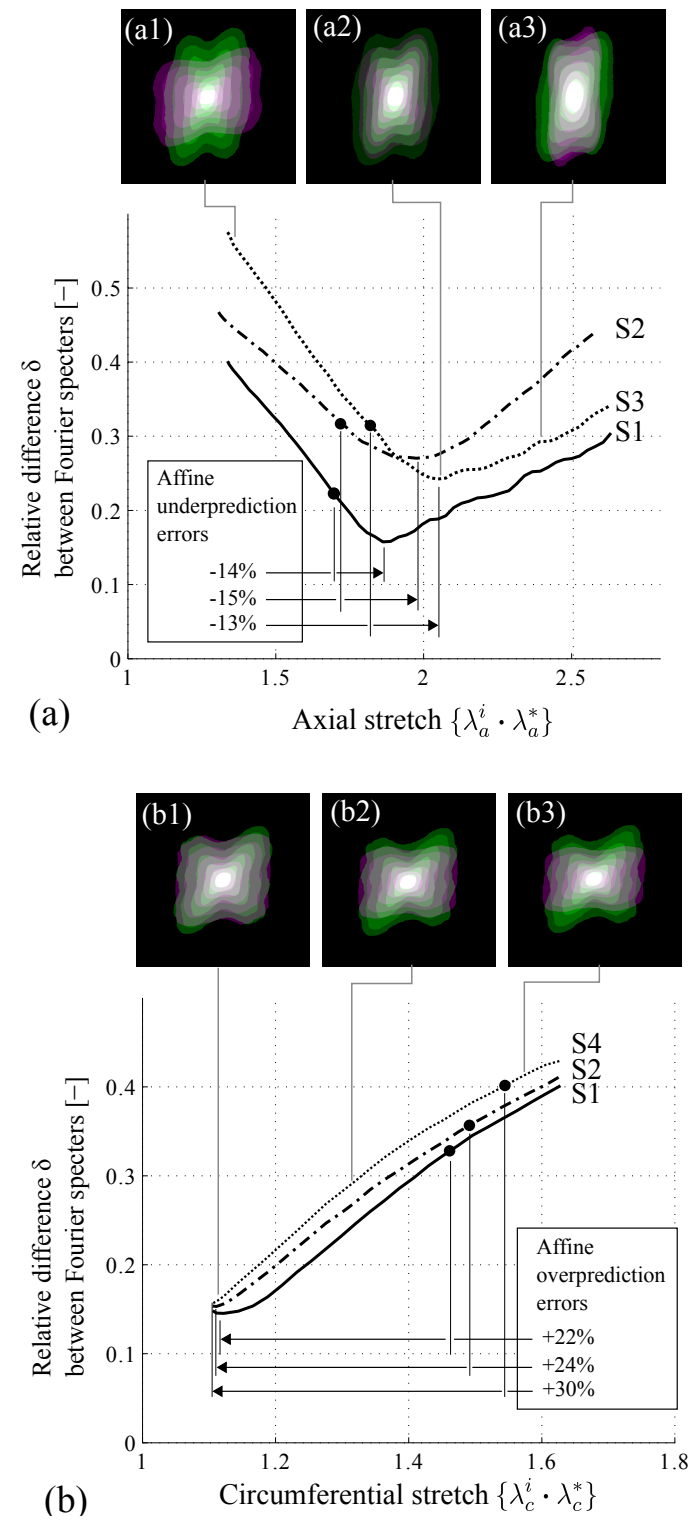

Fig. 5 Evolution of the relative difference $\delta$ between spectra corresponding to the numerically deformed morphology and the experimentally deformed morphology. (a) Loading scenario 1 (axial tension under constant imposed pressure): evolution of $\delta$ with the stretch numerically applied in the longitudinal direction $\lambda_{a}^{*}$; (b) Loading scenario 2 (inflation under constant axial stretch): evolution of $\delta$ with the stretch numerically applied in the circumferential direction $\lambda_{c}^{*}$. On each plot, the three curves refer to the three tested samples (either S1, S2, and S3 or S1, S2, and S4) and black dots • indicate the experimentally applied stretches $\lambda_{a}^{f}$ and $\lambda_{c}^{f}$. For each loading scenario, examples of superimposed spectra are provided for one carotid samples in (a1)-(a3) and (b1)-(b3) for different stretches numerically applied to the reference configuration. The purple (resp. green) spectrum corresponds to the numerically applied affine deformation (resp. to the experimentally applied loading). The difference between the experimental and the numerical stretches minimizing the difference between morphology spectra is represented by horizontal arrows in each figure. Accordingly, prediction error of affine kinematics are quantified under both loading scenarii. Prediction errors are negative when affine kinematics underpredicts the experimental stretch, and positive when affine kinematics overpredicts the experimental stretch. 


\section{Discussion}

The mechanically-induced reorientation of collagen fibers was the focus of several contributions, studying different collageneous tissues, such as tendon [32], collagenous constructs [10], skin [4,36], bovine pericardium [5], liver capsule [29], and carotid artery [31]. The debated question concerns the affine character of the reorientation process: do the fibers follow the strain imposed by their surrounding matrix, or are other mechanisms active in the reorientation process? The previously cited studies showed that collagen fibers present complex and potentially non-affine kinematics under load. However, while the reorientation of collagen fibers has been widely characterized, none of the contributions had investigated the effect of loading types on fiber kinematics while characterizing the affine character of the fiber reorientations. However, the results collected in the present paper suggest that the loading scenario, and more precisely the local strain tensor, plays an important role on bundle kinematics. We therefore propose to compare our results to the other available studies, paying a special attention to the loading scenario which was imposed to the arterial tissue.

In the present contribution, we investigated the kinematics of adventitial collagen bundles, when subjecting the arterial tissue to two different loading scenarii, namely axial tension under constant pressure and inflation under constant axial stretch, while the microscopic configuration of adventitial collagen was acquired by means of multiphoton microscopy. Regarding the first loading scenario, maintaining a constant pressure $(\mathrm{P}=100 \mathrm{mmHg})$ while applying axial stretches produced a pronounced reorientation of adventitial collagen fibers. To our best knowledge, the contribution the closest to ours is the one of Chen et al. (2013) [12], who studied the behavior of collagen fibers of coronary arteries under axial tensile loading while maintaining a constant circumferential stretch. In these tests, fiber reorientations showed limited reorientations (their rotation being $13 \%$ lower than predicted by the affine kinematics). The discrepancy with our results may originate in that, under such a deformation, no transverse strain of the sample is possible (through the 
use of a balloon catheter). In the present study however, the transverse strain observed while applying an axial tension to the samples S1; S2 and S3 was measured $15 \%, 18 \%$ and 18\%, respectively. As a result, the deformations applied by Chen et al. (2013) [12] induced a different strain state within the tissue as compared to our protocol. This may explain the different fiber kinematics.

We also investigated the fiber kinematics under inflation tests at a constant imposed axial strain. The applied methodology for the characterization of fiber kinematics suggests that inflating the artery at the constant in vivo length does not produce noticeable rotations of collagen fibers in the adventitia. Collagen fibers first straighten (engagement) and then keep their orientation unchanged. The same result was also obtained by Schrauwen et al. (2012) [44], who submitted carotid arteries to inflation after applying an initial stretch of $\lambda=1.5$. They measured fiber waviness and orientation across a given region of interest. Results showed that after unfolding (waviness parameter $>0.8$, corresponding to an applied pressure of 60 $\mathrm{mmHg}$ ), the peak orientation density did not vary further during the deformation (maximum pressure $120 \mathrm{mmHg}$ ), indicating that fibers did not reorient during deformation. Microscopy images also suggested, that the global orientations of the crimped fibers did not vary noticeably during the decrimping process occurring early in the inflation procedure (0 to $60 \mathrm{mmHg}$ ). Also, in the contribution of Chen et al. (2013) [12] and despite the different type of artery, adventitial collagen was shown to behave similarly to our results. Referring to the presented results [12] and interpreting fiber reorientations under increasing circumferential stretches $(\lambda$ $=1$ to $\lambda=1.7)$ at a given axial stretch $(\lambda=1.5)$, which is a loading scenario kinematically equivalent to inflation at constant axial stretch, we note that fibers showed limited reorientations (their rotation being 30\% lower than predicted by the affine kinematics). To our best knowledge, only Keyes et al. (2011) [30] obtained results with different trends although applying an analogous mechanical protocol. They found that adventitial collagen fibers reorient significantly from axial to circumferential directions under high pressure. These differences may be attributed 
to a different tissue type and a different methodology, given that a fixed region of interest was not tracked during the overall microscopy procedure of these authors.

The kinematics of collagen bundles are also modified when the applied loading scenario induces yet other macroscopic strains. Indeed, Wan et al. (2012) [52] compared three inflation configurations (increasing internal pressure) while simultaneously reducing axial stretch. They found that collagen in mouse adventitia shows affine kinematics in the physiological range of deformations. Furthermore, Sack (2003) [43] documented collagen kinematics under planar biaxial deformation with conclusions supporting the affine assumption for fiber kinematics, i.e. fibers following the macroscopic strain. In a previous study [31], we focused on uniaxial tensile tests of strips of carotid arteries, showing that collagen bundles reorient faster than predicted by affine kinematics. The discrepancies between the presented results and those of the latter studies most certainly lie in different mechanical protocols, inducing different strain paths.

The complex rearrangements of collagen fibers under macroscopic loading raise the question of the underlying mechanisms governing their complex kinematics. While fiber uncrimping is intrinsically a non-affine mechanism [16,34], we show in the present study that the non-affine character of collagen motion concerns also rigid motions of collagen fibers at the fiber lengthscale, regardless of its crimped or straight state. In order to explain the motion of collagen fibers, fiber-matrix interactions have to be taken into account. Previous studies have investigated the role of the non-collagenous matrix in the micromechanics of soft biological tissues, hence influencing collagen kinematics $[5,50]$. In particular, recent modelling results have suggested that the non-linear mechanical response of the tissue is due to structural effects (spin effects) of the fibers network in interaction with the surrounding matrix [37]. Presumably, a redistribution of microscopic stress through proteoglycans distributed in the matrix [50] influences fiber motion, with potential inner sliding inside each collagen fiber being responsible for the tissue's plastic response [36]. Besides, fiber-fiber interactions have been considered both in experimental 
investigations [13] as in modelling formulations [33,48,9,37]. While Lanir (1979)

[33] proposed a schematic representation of the collagen and elastin network structure in generalized collagenous tissues, with collagen undulation induced by the elastin exerting tension on them, Chow et al. (2014) [13] illustrated structural and mechanobiological interactions between fibers, eventually showing that, in porcine thoracic aortas, the elastic fibers are under tension and induce an intrinsic compressive stress on the collagen. From the modelling point of view, Stylioanopulos and Barocas (2007) [48] showed that a network composed of straight fibers interacting with each other via freely rotating crosslinks, incorporated as structural description of a Representative Volume Element in a multiscale model, provides good predictions of arterial biomechanics. Concerning the contribution of Morin et al. (2015) [37], the developed micromechanical model predicts the load-induced decrimping and rotations of the fibers and the subsequent stiffening of the mechanical behavior of a Representative Volume Element, accounting for both fiber-fiber interactions and fiber-matrix interactions, while introducing linear elastic constitutive behavior for fibers and matrix phases.

These observations, coupled to (i) the evidenced coexistence of collagen and elastin in the adventitial layer of arteries such as the rabbit carotid [31] or the pig coronary artery [12], and to (ii) findings about the load-bearing properties of collagen fibers (the elastic modulus of collagen fibers being greater than the elastic modulus of elastic fibers by a factor $10^{3}[54,6,17]$ ) and to (iii) the current understanding of both collagen-elastin fiber interactions and rearrangements [33, $13,18,53]$, suggest possible links with the cellular tensegrity theory $[28,47,46]$. The cellular tensegrity theory, developed for subcellular descriptions, proposes a representation of the cellular cytostructure through a network of high-strength microtubules, maintained in compression by a network of low-strength actin fibers under tension, the whole system being in static equilibrium in the absence of an external load. Such theory can be extended to collagenous networks as shown in Fig. 6, where a simplified tensegrity model inspired from Luo et al. (2008) 
[35] is introduced to represent arterial adventitia with rigid collagen fibers and compliant links between the collagen fibers [13]. Such a model may explain the results observed in the present study: under inflation loading at a constant axial stretch, fiber rotation is impossible, as it would imply a transverse strain, the fibers being rigid. Conversely, under axial tensile loading at a fixed inner pressure, fiber rotation is the only way to allow the extension. Noticeably, this model may also explain the results of Chen et al. (2013) [12]; namely when applying an axial tension while maintaining the circumferential stretch constant (hence disabling transverse strain), fiber rotations were limited and overpredicted by affine predictions. All in all, this representation of the adventitial microstructure, based on a previously applied tensegrity model [35], could explain the complex kinematics of collagen fibers and their dependence on the type of loading scenario that we observed in the present study.

Still, several limitations to this study should be mentioned. In fact, the deformations undergone by the arterial samples match only partially the in vivo mechanical loading. This is obviously the case of axial tension under constant pressure, but also to a certain extent of inflation at a fixed in vivo stretch. Also, the test temperature was maintained at $20^{\circ} \mathrm{C}$, while it is $38-40^{\circ} \mathrm{C}$ in vivo, and the deformation was applied under quasi static conditions, which cannot render the dynamic nature of the pulsatile loading in vivo (of the order of $1 \mathrm{~Hz}$ ). Moreover, the applied range of pressure did not match the in vivo amplitude between systole and diastole, which ranges between $105 \pm 15$ and $125 \pm 15 \mathrm{mmHg}$ [20] in the rabbit artery, Besides, excision of the arterial tissue from its native environment initiated, prior to testing, the destructive action of degradative enzymes affecting collagen content. In the meantime, as the tissue was stored in PBS, it was prone to a modification of residual stress, as a result of the evolving fixed-charge density accumulated by proteoglycans [3], eventually causing a swelling of the tissue. As a result, it cannot be neglected that this residual stress, resulting from sample storing in PBS, influences both the mechanical response and collagen fiber kine- 


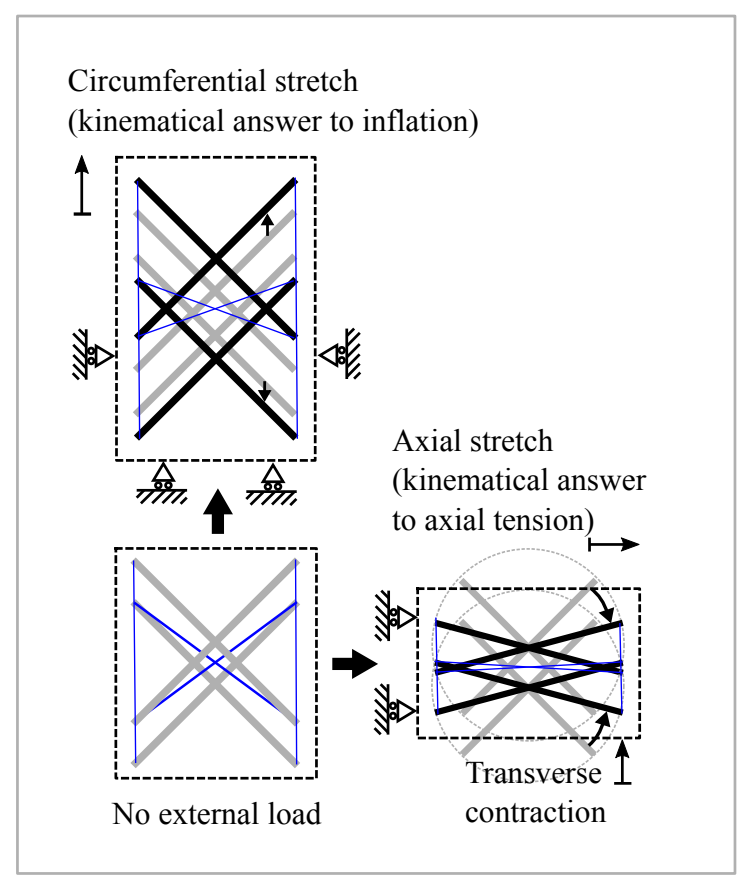

Fig. 6 Schematic representation of adventitial microstructure inspired from the tensegrity structure of Luo et al 2008 [35]. Black segments represent here low-compliant collagen bundles, while blue segments represent high-compliant binding fibers which exert compressive forces on the collagen bundles under zero load. The two considered loading scenarii are represented, characterized by free transverse strain boundary condition in the case of scenario 1 (axial tension), and by fixed axial length boundary condition in the case of scenario 2 (inflation). The grey segments represent the initial positions of collagen bundles, before deformation, to be compared with final positions in black, after deformation.

matics. However, the objective of our contribution was to investigate a possible dependence of fiber kinematics to different loading scenarii, rather than to characterize the fiber kinematics under in vivo conditions. As a result, the choice of test temperature and of pressure range did not impair our observations and conclusions. Concerning loading dynamics, the quasi-static deformation protocol with tissue preconditioning allowed live imaging under multiphoton microscopy with repeatable results, which would not be possible if a dynamic pulsatile loading would have been applied as it is the case in vivo. Another limitation concerns the application of a Maximum Intensity Projection (MIP) algorithm (see section 2.5) to the 3D images stack and the resulting 2D morphology that we analyzed. Indeed, fibers localized on the most outer circumferential layers of the artery appeared the 
brightest and uninterrupted in the resulting 2D morphology. As for deeper fibers, they appeared eventually overlapped by the outer fibers, with a decreased contrast on the image. As a result, although they are identical from the biological point of view, the different fibers did not contribute to the overall image spectrum with the same weight. This limitation highlights the importance of following the same region of interest during the microscopy procedure, given that the discrepancy of contributions relative to the different fibers with regards to the resulting $2 \mathrm{D}$ spectrum is identical in the different compared projected (MIP) morphologies. Finally, a limitation originates in the spectral comparison between, on the one hand, a morphology characterized by a slight crimping of fiber, and on the other hand, a morphology characterized by fully engaged, straight fibers. In fact, when choosing the reference morphology for spectrum analysis, a part of the computed spectrum difference $\delta$ originates in the undulated character of the fibers, and another part originates in the different fiber orientations at the fiber bundle scale. A preliminary analysis of spectrum sensitivity, using fictitious collagen images, showed that the undulation contribution to the computed spectrum difference was negligible with regards to the orientation contribution, provided that the small lengthscales corresponding to fiber crimping were excluded from our analysis. However, this is not anymore true when fiber crimping is important, hindering an analysis of global bundle orientation (as it is the case in the unloaded configurations in Krasny et al. (2017) [31]). This is the major reason, why the reference configurations of the loading scenarii were chosen to induce a partial loading $\left(\lambda_{a}=1.3, \mathrm{P}=100 \mathrm{mmHg}\right.$ for scenario $1, \lambda_{a}=1.6, \mathrm{P}=20 \mathrm{mmHg}$ for scenario 2 ): in fact the fibers appeared close to engagement or partially engaged. Another reason for this choice of loading originated in the need to prevent the potential buckling of the samples at high pressure and low axial stretch [22], which would have disabled the analysis of macroscopic and microscopic kinematics. As concerns the interpretation of the adventitial microstructure using a tensegrity model, it must be mentioned that, at this stage of knowledge about the arterial microstructure, its physical validity 
is conditioned by the verification of several assumptions. In particular, collagen bundles are not capable of supporting the compressive forces exerted by elastin (which results in their crimped state under zero external load). However, this role in the overall force equilibrium may be played by proteoglycans: due to their ability to trap water molecules along with the sodium ions that equilibrate their electric charge [3,7], the hydrated proteoglycans provide a mechanical resistance to compression. Another assumption concerns the nature of the bindings between collagen and elastin, which should be characterized experimentally in the future.

Nevertheless, the original outcomes of the present study significantly contribute to a better understanding of arterial kinematics at the microscopic scale. From the experimental side, future work should characterize the interactions between collagen and other binding proteins such as elastin. From the modeling side, tensegrity models could represent arterial microstructure and its associated mechanics, and future work should further test their applicability at the tissue level with collagen, elastin and proteoglycans as principal components (Fig. 6).

Acknowledgements This work was supported by the ARC 2 "Bien-être et vieillissement" research program of the Auvergne-Rhône-Alpes region (FR), with financial contributions from ERC consolidator grant "Biolochanics", Research group MMV - Mécanique des Matériaux du Vivant (LTDS, CNRS UMR 5513), and the Institut Carnot Ingénierie@Lyon (I@L). The authors thank Prof. Eric Viguier (VetAgro Sup, Université de Lyon, FR), Dr. Caroline Boulocher (VetAgro Sup, Université de Lyon, FR) and Mr. Fabrice Desplanches (Centre Lago, Vonnas, FR) for their help in provision and excision of arterial specimen. Authors also thank the IVTV (ANR-10-EQPX-06-01) team and especially Ing. Ophélie Pollet for her support during the imaging process, as well as Dr. Damien Constant (Ecole Centrale Lyon, FR) for their technical contribution in the preparation of the mechanical setup.

\section{References}

1. Arkill, K.P., Moger, J., Winlove, C.P.: The structure and mechanical properties of collecting lymphatic vessels: an investigation using multimodal nonlinear microscopy. Journal of Anatomy 216(5), 547-555 (2010) 
2. Ayres, C., Bowlin, G.L., Henderson, S.C., Taylor, L., Shultz, J., Alexander, J., Telemeco, T.A., Simpson, D.G.: Modulation of anisotropy in electrospun tissue-engineering scaffolds: Analysis of fiber alignment by the fast Fourier transform. Biomaterials 27(32), 5524-5534 (2006)

3. Azeloglu, E.U., Albro, M.B., Thimmappa, V.A., Ateshian, G.A., Costa, K.D.: Heterogeneous transmural proteoglycan distribution provides a mechanism for regulating residual stresses in the aorta. American Journal of Physiology-Heart and Circulatory Physiology 294(3), H1197-H1205 (2008)

4. Bancelin, S., Lynch, B., Bonod-Bidaud, C., Ducourthial, G., Psilodimitrakopoulos, S., Dokládal, P., Allain, J.M., Schanne-Klein, M.C., Ruggiero, F.: Ex vivo multiscale quantitation of skin biomechanics in wild-type and genetically-modified mice using multiphoton microscopy. Scientific reports 5, 17,635 (2015)

5. Billiar, K., Sacks, M.: A method to quantify the fiber kinematics of planar tissues under biaxial stretch. Journal of Biomechanics 30(7), 753-756 (1997)

6. Burton, A.C.: Relation of structure to function of the tissues of the wall of blood vessels. Physiological Reviews 34(4), 619-642 (1954)

7. Buschmann, M., Grodzinsky, A., et al.: A molecular model of proteoglycan-associated electrostatic forces in cartilage mechanics. J Biomech Eng 117(2), 179-92 (1995)

8. Canham, P.B., Whittaker, P., Barwick, S.E., Schwab, M.E.: Effect of pressure on circumferential order of adventitial collagen in human brain arteries. Canadian journal of physiology and pharmacology 70(2), 296-305 (1992)

9. Cardamone, L., Valentin, A., Eberth, J., Humphrey, J.: Origin of axial prestretch and residual stress in arteries. Biomechanics and modeling in mechanobiology 8(6), 431-446 (2009)

10. Chandran, P.L., Barocas, V.H.: Affine versus non-affine fibril kinematics in collagen networks: theoretical studies of network behavior. Journal of Biomechanical Engineering 128(2), 259-70 (2006)

11. Chen, H., Liu, Y., Slipchenko, M.N., Zhao, X., Cheng, J.X., Kassab, G.S.: The layered structure of coronary adventitia under mechanical load. Biophysical Journal 101(11), 2555-2562 (2011)

12. Chen, H., Slipchenko, M.N., Liu, Y., Zhao, X., Cheng, J.X., Lanir, Y., Kassab, G.S.: Biaxial deformation of collagen and elastin fibers in coronary adventitia. Journal of Applied Physiology 115(11), 1683-1693 (2013)

13. Chow, M.J., Turcotte, R., Lin, C.P., Zhang, Y.: Arterial extracellular matrix: a mechanobiological study of the contributions and interactions of elastin and collagen. Biophysical Journal 106(12), 2684-2692 (2014) 
14. D'Amore, A., Stella, J.A., Wagner, W.R., Sacks, M.S.: Characterization of the complete fiber network topology of planar fibrous tissues and scaffolds. Biomaterials 31(20), 5345$5354(2010)$

15. Dingemans, K.P., Teeling, P., Lagendijk, J.H., Becker, A.E.: Extracellular matrix of the human aortic media: an ultrastructural histochemical and immunohistochemical study of the adult aortic media. The Anatomical Record 258(1), 1-14 (2000)

16. Fan, R., Sacks, M.S.: Simulation of planar soft tissues using a structural constitutive model: finite element implementation and validation. Journal of Biomechanics 47(9), 2043-2054 (2014)

17. Faury, G.: Function-structure relationship of elastic arteries in evolution: from microfibrils to elastin and elastic fibres. Pathologie Biologie 49(4), 310-325 (2001)

18. Ferruzzi, J., Vorp, D., Humphrey, J.D.: On constitutive descriptors of the biaxial mechanical behaviour of human abdominal aorta and aneurysms. Journal of the Royal Society, Interface 8(56), 435-450 (2011)

19. Gasser, T.C., Holzapfel, G.A.: Finite element modeling of balloon angioplasty by considering overstretch of remnant non-diseased tissues in lesions. Computational Mechanics 40(1), 47-60 (2007)

20. Govyrin, V.: Measurement of arterial pressures in intact rabbits. Bulletin of Experimental Biology and Medicine 44(1), 896-897 (1957)

21. Greenwald, S., Moore, J., Rachev, A., Kane, T., Meister, J.J.: Experimental investigation of the distribution of residual strains in the artery wall. Journal of Biomechanical Engineering 119(4), 438-444 (1997)

22. Han, H.C., Chesnutt, J.K., Garcia, J.R., Liu, Q., Wen, Q.: Artery buckling: new phenotypes, models, and applications. Annals of Biomedical Engineering 41(7), 1399-1410 (2013)

23. Hill, M.R., Duan, X., Gibson, G.A., Watkins, S., Robertson, A.M.: A theoretical and nondestructive experimental approach for direct inclusion of measured collagen orientation and recruitment into mechanical models of the artery wall. Journal of Biomechanics 45(5), $762-771(2012)$

24. Holzapfel, G.A., Gasser, T.C.: A viscoelastic model for fiber-reinforced composites at finite strains: Continuum basis, computational aspects and applications. Computer Methods in Applied Mechanics and Engineering 190(34), 4379-4403 (2001)

25. Holzapfel, G.A., Sommer, G., Gasser, C.T., Regitnig, P.: Determination of layer-specific mechanical properties of human coronary arteries with nonatherosclerotic intimal thickening and related constitutive modeling. American Journal of Physiology-Heart and Circulatory Physiology 289(5), H2048-H2058 (2005)

26. Humphrey, J.: Cardiovascular Solid Mechanics: Cells, Tissues, and Organs. Springer (2002) 
27. Humphrey, J., Canham, P.: Structure, mechanical properties, and mechanics of intracranial saccular aneurysms. Journal of Elasticity and the Physical Science of Solids 61(1-3), 49-81 (2000)

28. Ingber, D.E., et al.: Cellular tensegrity: defining new rules of biological design that govern the cytoskeleton. Journal of Cell Science 104, 613-613 (1993)

29. Jayyosi, C., Coret, M., Bruyere-Garnier, K.: Characterizing liver capsule microstructure via in situ bulge test coupled with multiphoton imaging. Journal of the Mechanical Behavior of Biomedical Materials 54, 229-243 (2016)

30. Keyes, J.T., Borowicz, S.M., Rader, J.H., Utzinger, U., Azhar, M., Geest, J.P.V.: Design and demonstration of a microbiaxial optomechanical device for multiscale characterization of soft biological tissues with two-photon microscopy. Microscopy and Microanalysis 17(02), 167-175 (2011)

31. Krasny, W., Morin, C., Magoariec, H., Avril, S.: A comprehensive study of layer-specific morphological changes in the microstructure of carotid arteries under uniaxial load. Acta Biomaterialia - manuscript accepted 0(00), 00-00 (2017)

32. Lake, S.P., Hadi, M.F., Lai, V.K., Barocas, V.H.: Mechanics of a fiber network within a non-fibrillar matrix: model and comparison with collagen-agarose co-gels. Annals of Biomedical Engineering 40(10), 2111-2121 (2012)

33. Lanir, Y.: A structural theory for the homogeneous biaxial stress-strain relationships in flat collagenous tissues. Journal of biomechanics 12(6), 423-436 (1979)

34. Lee, C.H., Zhang, W., Liao, J., Carruthers, C.A., Sacks, J.I., Sacks, M.S.: On the presence of affine fibril and fiber kinematics in the mitral valve anterior leaflet. Biophysical journal 108(8), 2074-2087 (2015)

35. Luo, Y., Xu, X., Lele, T., Kumar, S., Ingber, D.E.: A multi-modular tensegrity model of an actin stress fiber. Journal of Biomechanics 41(11), 2379-2387 (2008)

36. Lynch, B., Bancelin, S., Bonod-Bidaud, C., Gueusquin, J.B., Ruggiero, F., Schanne-Klein, M.C., Allain, J.M.: A novel microstructural interpretation for the biomechanics of mouse skin derived from multiscale characterization. Acta Biomaterialia 50, 302-311 (2017)

37. Morin, C., Avril, S., Hellmich, C.: The fiber reorientation problem revisited in the context of Eshelbian micromechanics: theory and computations. Pamm 15(1), 39-42 (2015)

38. Morrill, E.E., Tulepbergenov, A.N., Stender, C.J., Lamichhane, R., Brown, R.J., Lujan, T.J.: A validated software application to measure fiber organization in soft tissue. Biomechanics and Modeling in Mechanobiology 15(6), 1467-1478 (2016)

39. O’Connell, M.K., Murthy, S., Phan, S., Xu, C., Buchanan, J., Spilker, R., Dalman, R.L., Zarins, C.K., Denk, W., Taylor, C.A.: The three-dimensional micro- and nanostructure of the aortic medial lamellar unit measured using 3D confocal and electron microscopy imaging. Matrix Biology 27(3), 171-181 (2008) 
40. Polzer, S., Gasser, T.C., Forsell, C., Druckmüllerova, H., Tichy, M., Staffa, R., Vlachovsky, R., Bursa, J.: Automatic identification and validation of planar collagen organization in the aorta wall with application to abdominal aortic aneurysm. Microscopy and Microanalysis 19(06), 1395-1404 (2013)

41. Rezakhaniha, R., Agianniotis, A., Schrauwen, J.T.C., Griffa, A., Sage, D., Bouten, C.V.C., Van De Vosse, F.N., Unser, M., Stergiopulos, N.: Experimental investigation of collagen waviness and orientation in the arterial adventitia using confocal laser scanning microscopy. Biomechanics and Modeling in Mechanobiology 11(3-4), 461-473 (2012)

42. Roy, S., Boss, C., Rezakhaniha, R., Stergiopulos, N.: Experimental characterization of the distribution of collagen fiber recruitment. Journal of Biorheology 24(2), 84-93 (2011)

43. Sacks, M.S.: Incorporation of experimentally-derived fiber orientation into a structural constitutive model for planar collagenous tissues. Journal of Biomechanical Engineering 125(2), 280-287 (2003)

44. Schrauwen, J.T.C., Vilanova, A., Rezakhaniha, R., Stergiopulos, N., van de Vosse, F.N., Bovendeerd, P.H.M.: A method for the quantification of the pressure dependent 3D collagen configuration in the arterial adventitia. Journal of Structural Biology 180(2), 335-342 (2012)

45. Schriefl, J., Reinisch, J., Sankaran, S., Pierce, D.M., Holzapfel, G.A.: Quantitative assessment of collagen fibre orientations from two-dimensional images of soft biological tissues. Journal of The Royal Society Interface 9, 3081-3093 (2012)

46. Stamenovic, D., Coughlin, M.F.: A quantitative model of cellular elasticity based on tensegrity. Journal of Biomedical Engineering 122(1), 39-43 (2000)

47. Stamenović, D., Fredberg, J.J., Wang, N., Butler, J.P., Ingber, D.E.: A microstructural approach to cytoskeletal mechanics based on tensegrity. Journal of Theoretical Biology 181(2), 125-136 (1996)

48. Stylianopoulos, T., Barocas, V.H.: Multiscale, structure-based modeling for the elastic mechanical behavior of arterial walls. Journal of Biomechanical Engineering 129(4), 611$618(2007)$

49. Sugita, S., Matsumoto, T.: Multiphoton microscopy observations of 3D elastin and collagen fiber microstructure changes during pressurization in aortic media. Biomechanics and Modeling in Mechanobiology 16(3), 763-773 (2017)

50. Thorpe, C.T., Birch, H.L., Clegg, P.D., Screen, H.R.: The role of the non-collagenous matrix in tendon function. International Journal of Experimental Pathology 94(4), 248$259(2013)$

51. Van Zandvoort, M., Engels, W., Douma, K., Beckers, L., Oude Egbrink, M., Daemen, M., Slaaf, D.W.: Two-photon microscopy for imaging of the (atherosclerotic) vascular wall: A proof of concept study. Journal of Vascular Research 41(1), 54-63 (2004) 
52. Wan, W., Dixon, J.B., Gleason, R.L.: Constitutive modeling of mouse carotid arteries using experimentally measured microstructural parameters. Biophysical Journal 102(12), 2916-2925 (2012)

53. Wang, R., Brewster, L.P., Gleason, R.L.: In-situ characterization of the uncrimping process of arterial collagen fibers using two-photon confocal microscopy and digital image correlation. Journal of Biomechanics 46(15), 2726-2729 (2013)

54. Wenger, M.P., Bozec, L., Horton, M.A., Mesquida, P.: Mechanical properties of collagen fibrils. Biophysical Journal 93(4), 1255-1263 (2007)

55. Zeinali-Davarani, S., Wang, Y., Chow, M.J., Turcotte, R., Zhang, Y.: Contribution of collagen fiber undulation to regional biomechanical properties along porcine thoracic aorta. Journal of Biomechanical Engineering 137(5), 051,001 (2015)

56. Zhang, L., Lake, S., Barocas, V., Shephard, M., Picu, R.: Cross-linked fiber network embedded in an elastic matrix. Soft Matter 9(28), 6398-6405 (2013)

57. Zisserman, A.: Image Analysis course - 2D Fourier transforms and applications (2014)

58. Zulliger, M.A., Fridez, P., Hayashi, K., Stergiopulos, N.: A strain energy function for arteries accounting for wall composition and structure. Journal of Biomechanics 37(7), 989-1000 (2004) 